\title{
Application of Cable with Insulating and Fire-retardant Coating
}

\author{
Wang Ling \\ Benxi Power Supply Company of National Grid Liaoning Electric Power Co., Ltd. \\ bxwl_650928@126.com
}

Keywords: fire-resistant; flame retardant; optical cable

\begin{abstract}
With the increasing need for flame-retardant optical cable currently, requirements for flame-retardant levels of optical cable differ in various occasions. This paper introduces the differences between optical cables with different flame-retardant levels, focuses on describing the design of a fire-resistant and flame-retardant optical cable and then makes an experimental verification on its fire-resistant property.
\end{abstract}

\section{Introduction}

Traditional PVC sheath is poor in moisture-proof performance and not suitable for outdoor uses despite its incombustibility. According to reports, foreign countries have developed a dual-purpose drop optical cable or under-rod optical cable that is resistant to low outdoor temperature and UV radiation and at the same time flame-retardant and with wiring easy to bend. This optical cable is made of PVC tight tube fiber, water-blocking absorbent powder and low-smoke halogen-free and flame-retardant jacket. Currently relevant domestic researches are still in a backward position. One in the event of fire, the flame-resistance of traditional optical cable is not ideal enough to control the fire effectively.

Due to the poor flame-resistance, moisture-proof performance and uneasy wiring-bend property of optical cable itself, in this research, a thin BCN coating is sprayed on the optical cable by spraying technology. The BCN coating is resistant to acid and corrosion and more importantly, is excellent in insulting and fire-proof performance, which is essential for the fire-proof protective layer of the optical cable.

\section{Difference in the Flame-retardant Optical Cables with different Levels}

As the tradition of optical fiber and cable industry, fire retardant, low smoke halogen free (LSOH) or low smoke fume (LSF), fire resistant and others with certain fire-proof property are called collectively fire-proof optical cable. The differences between them are as follows:

\section{Flame Retardant Optical Cable.}

Fire-retardant optical cable is characterized by delaying the spread of flame along with the cable line. The low cost makes it the widely adopted type of fire-proof optical cable. No matter it is a single cable or cable with multiple layers, optical cable can help keep flame spread within certain range and therefore prevent major disasters caused by flame spread and improve the fire-proof capacity of optical cable line.

\section{Low-smoke Halogen-free Flame-retardant Optical Cable.}

Low-smoke halogen-free flame-retardant optical cable is characterized by its excellent flame retardant property and the non-halogen material. The cable is less corrosive and toxic when burning and produces little smoke, thus reducing damages on human body, instruments and equipment and contributing to the timely rescue of fire. Low-smoke halogen-free flame-retardant optical cable has a poorer mechanical property than common cables despite its excellent flame resistance, corrosion resistance and low smoke concentration.

\section{Low Smoke Fume Flame-retardant Optical Cable.}

Low smoke fume flame-retardant optical cable has a hydrogen chloride emission and smoke density indicator between flame-retardant optical cable and low-smoke halogen-free flame-retardant optical cable. Low halogen cable also contains materials with halogen but at a lower level. This 
cable is characterized by its flame-retardant property and also less release of smoke and lower emission of hydrogen chloride during combustion. Low smoke fume flame-retardant optical cable is generally made from polyvinyl chloride (PVC) as the substrate and efficient fire retardant, HCL absorber and smoke suppressant as the subsidiaries.

\section{Fire Resistant Flame Retardant Optical Cable.}

Fire resistant optical cable can keep functioning for a certain period of time and maintain the circuit integrity when fire occurs. Fire resistant flame retardant optical cable produces less sour gas and smoke during combustion that greatly improves the fire resistant flame retardant property. What's more, the cable can maintain the circuit integrity along with water spray and mechanical vibration in combustion.

\section{Burning Behavior and Flame Retardant Mode of Optical Cable Materials}

\section{Burning Behavior of Optical Cable Materials.}

Burning behavior of optical cable materials can be described by the following several performance parameters: a) degree of difficulty in igniting the material, which can be defined by critical oxygen index COI or limiting oxygen index LOI. The higher the value, the more difficult for the material to get ignited and flame spread. Typically, COI $\geq 30 \%$ indicates that it is flame retardant; $\mathrm{LOI} \leq 23 \%$ indicates that it's flammable; LOI between $24 \%-28 \%$ indicates that it is slightly flame retardant; LOI between $9 \%-35 \%$ indicates that it is flame retardant; and LOI $\geq 36 \%$ indicates that it is perfectly flame retardant. b) The speed of flame spread on the surface of the material. c) Flame resistance, i.e. the speed of fire burning through the material. d) Heat release rate HRR. e) Degree of difficulty in extinguishing the fire. f) Smoke resistance, including smoke amount, smoking speed and composition of the smoke. g) Composition, amount and generating speed of toxic gases produced.

\section{Flame Retardant Mode of Optical Cable Materials.}

Chemical or physical method can be used to change the structure of polymer and thus achieve flame retardant effect for optical cable. For example, add flame retardant elements in the polymer molecule, such as bromine, chlorine, phosphorus, antimony, boron and so on, and adopt chemical crosslinking or radiation crosslinking to change the linear polymer molecules into a three-dimensional material structure to improve its thermal stability and char formation. Or add flame retardants to the matrix polymer and during combustion, the flame-retardant component will function in different regions of polymer combustion in different ways and mechanisms. Flame retardants can function in the following several ways:

Gas-phase flame retardant achieves the flame retardant effect by radicals inhibiting chain-propagating spread of polymer combustion reaction in the gas phase. Flame retardants can capture radicals within the combustion zone in the gas phase and thus prevent the spread of flame, reduce the flame density in the combustion zone and finally gradually reduce the combustion reaction rate until terminated.

Condensed phase flame retardant achieves the flame retardant effect by inhibiting thermal decomposition of the polymer and prevent release of flammable gas in the solid phase. Flame retardants at high temperatures can form molten glassy materials or a foam char layer to cover the surface of polymer to insulate heat and oxygen, preventing flammable gas from escaping outward and thus achieving the flame retardant purpose.

Interrupting heat exchange achieves the flame retardant effect by taking away heat generated by polymer instead of allowing them to stay decomposing the polymer. Flame retardants at high temperatures can make heat-absorbing reactions to adsorb parts of the heat generated by combustion and thus reduce the surface temperature of flammable materials, inhibit effectively the generation of flammable gases and prevent flames from spreading. Flame retardants under heat generates inflammable gases that can dilute the flammable gases decomposed by flammable materials, and thus reduces the flammable gases concentration under ignition limit and at the same time reduces the oxygen concentration in the combustion zone to inhibit the combustion and achieve the flame retardant effect. 
Charring effect achieves the flame retardant effect by forming chars in the decomposition of polymer that can reduce volatile generation and cover the surface of polymer to insulate it with flames and to make it more difficult for further heat decomposition. In addition, the char layer can also prevent the internal heat decomposition from entering the gas phase and combustion. Generally, every $1 / 3$ increase of char formation can bring in a $1 / 2$ reduction of smoke generation. In order to achieve a flame retardant level of UL94 and V20, amount of chars formed should be at least 30\%.

Synergistic effect means that the combined effect of all components is greater than the total effect of each component. The most typical synergistic effect is antimony 2 halogen synergies, where antimony oxide in its common form Sb2O3 is used with chlorine or bromine-containing flame retardants. During the gas phase, antimony oxide and halogen generate antimony tri-halide; as the inhibitor for flames, antimony tri-halide can capture radicals like H. , HO in the flame; evaporated antimony tri-halide can stay for a long time in the combustion zone and dilute the flammable gases, and at the same time cover the surface of polymer to insulate heat and reduces the temperature and speed for polymer decomposition; the formed char layer wrap up the polymer and prevent flammable gases from leaking. Some antimony halides can be used as the carbon catalyst and capturer of radicals on the surface in the condensed phase. There are other synergies, such as antimony oxide Đlon-halogen synergy, phosphorous - halogen synergy, nitrogen - halogen synergy, phosphorus - phosphorus synergy and so on.

\section{Structure Design of Fire Resistant and Flame Retardant Optical Cable}

Since the electricity and similar industries impose rather high requirements on the fire resistant and flame retardant properties of optical cable, the designed optical cable structure should possess such properties as halogen free, low smoke, low toxicity, fire-resistant and flame-retardant. Technical specifications of the designed fire resistant and flame retardant optical cable should meet the YD / T 1114-2001 halogen free flame retardant optical cable standard. Core of optical cable should not be filled with flammable materials. Insert single-mode or multimode fiber into a loose tube made of plastics with high modulus and fill the tuber with water blocking compounds. In the center of the core is a phosphate; the loose tube (or filled rope) around the center and the strengthened core are twisted into a compact circular cable core in a structure of water blocking yarns and blocks; external cable core should be wrapped up with mica tapes, flame retardant fiberglass tapes and flame retardant inner sheath; the external sheath should be a combination of steel strips and flame retardant outer sheath. The structured fire resistant and flame retardant optical cable is no specialty in production technology with common flame retardant optical cable, which will not be discussed in this paper. The key lies in the choose of flame retardant materials, including mica tapes, flame retardant fiberglass tape, flame retardant inner sheath and flame retardant outer sheath.

\section{Experimental Verification of the Fire Resistant and Flame Retardant Optical Cable}

Fire resistant test is carried out by the method specified in IEC60331-11 and IEC60331-25.

(1) The test should be done in an appropriate case without ventilation devices or devices that can generate toxic gas when burned. The outside temperature of the test case should be $5{ }^{\circ} \mathrm{C} \sim$ $40{ }^{\circ} \mathrm{C}$; (2) The sample should be a finished cable with a length of at least 5 meters that is horizontally placed with both ends extending outside; (3) The optical fiber should be connected to the optical cable test instrument to monitor / measure changes in light transmission under a predetermined wavelength; (4) The fire lasts for 90 minutes.

Process of Optical Cable Fire Resistant Test.

Before the fire resistant test, the wavelength loss of an optical fiber at a length of $155 \mathrm{~nm}$ is $0.201 \mathrm{~dB}$ and $0.225 \mathrm{~dB}$ after being burned for 90 minutes and cooled for 15 minutes, which is satisfactory. 


\section{Conclusion}

By structure design, product realization, fire resistance and tensile performance tests of a fire resistant and flame retardant optical cable, it can be concluded that the developed fire resistant and flame retardant optical cable in this structure is successful. As foreign and domestic users show, the design meets the requirements of users. As the society progresses, optical cable producers will continue to research on optical cable with special fire resistant and flame retardant properties and develop fire resistant optical cable with different structures to meet the requirements of more special occasions on fire resistant and flame retardant optical cable.

\section{Reference}

[1] Chen Dongliang, Bian Fuli. Researches on Fire Blocking Materials for Thermal Expansion Optical Cable[J]. Fire Technique and Products Information, 2003, (3): 25-26.

[2] Liu Liwen. New Technology of Fire Prevention for Optical Cable[J]. Construction Technology, 1999. (5): 57.

[3] Tao Jun. Application of Flame Retardant Optical Cable in Engineering Design [J]. Information Development and Economy, 2004, (6): 165-166.

[4] Wei Xuehai. Design and Development and Selected Application of Flame-retardant Fire-resistant Optical Cable [J]. Wire and Cable, 2005, (2): 43-45.

[5] Liu Feng. Flame Prevention and Toxic Gas Elimination - on the Ventilating Fire Resistant of Optical Cable [J]. Intelligent Building and City Information, 2004, (3): 67.69. 